\title{
3D-WAVELET TRANSFORM BASED SKIN CANCER CLASSIFICATION OF VGG-16 NETWORK MODEL
}

\author{
S.P Maniraj \\ Research Scholar, Department of Computer Science \& Engineering, School of Computing, \\ Sathyabama Institute of Science and Technology, Chennai, Tamilnadu, India - 600119. \\ spmaniraj1986@gmail.com, \\ P. Sardar Maran \\ Associate Professor, Department of Computer Science \& Engineering, School of Computing, \\ Sathyabama Institute of Science and Technology, Chennai, Tamilnadu, India - 600119. \\ psmaran@sathyabama.ac.in
}

\begin{abstract}
The RGB dermoscopic skin images utilize the Deep Convolutional Neural Network (DCNN) model with the wavelet analysis of skin image transformations. This proposed Computer-Aided Diagnosis (CAD) based early diagnosis of malignant melanoma skin cancer image has three phases. In this, the acquired image utilizes the median filter to remove noise and skin hairs in the initial phase of pre-processing stage. The second phase employs the 3-DimensionalDiscrete Wavelet Transformation (3D-DWT) feature extraction method with Transfer Learning (TL) technique. Here the 2D-DWT uses the grayscale image and 3D-DWT uses the RGB color image as input for feature extraction, and transformation performancesare evaluated and compared. The result obtained shows that the 3D-DWT-based skin image transformation with $\mathbf{9 7 \%}$ accuracy, $\mathbf{9 5 . 4 2 \%}$ sensitivity, and $\mathbf{9 7 . 7 8 \%}$ specificityprovides a high-resolution transformation of images. Hence, 3D-DWT transformations provide efficient feature datasets and CNNbased VGG-16 network classifiers for melanoma skin classification.
\end{abstract}

Keywords: Computer-Aided Diagnosis, dermoscopic images, Discrete Wavelet Transformation, Transfer Learning.

\section{Introduction}

A worldwide mortality rate is increased, as melanoma skin cancer is the most hazardous and fatal disease. A handheld instrument, the direct dermoscopic image is used for routine consultation to visualize problematic skin regions. However, all objective analysis, observations, and measurement of characteristics need for a substantial period. Therefore, for further examination, a permanent photographic record is generated termed a dermoscopic image. A great number of diagnostic information is gathered through dermoscopic images, and this is the most suitable approach for diagnosing skin cancer. Abnormal skin cell development is referred to as skin cancer that are classified as non-melanoma and melanoma cancer. Highly suspected skin lesions that don't treat fast may result in cancer disease. Dermoscopic skin lesion images with CNN aids in diagnosing and classify the non-cancerous tumor from the cancerous tumor as benign or malignant skin images. Melanoma is the malignant skin images that are the most life-threatening and harmful skin cancer. In the past few years, Deep learning neural network has significant advancements in the Medical Image processing technique that helps doctors diagnose and detect diseases faster and earlier.

Less time-consuming CAD technology is required as the medical field expects efficient and reliable techniques to diagnose these life-threatening diseases that cause high mortality globally for patients. The sample digital skin images are acquired from the Medical Repository $\mathrm{PH}^{2}$ Database as normal and malignant melanoma skinimages in this research.

\section{Literature Survey}

Diagnosis of melanoma, a deadly cancer disease utilizing the dermoscopic skin lesion image, employs DCNN (Acosta et al., 2021). This automated framework initially aids the masking technique to crop the ROI region of test images. The second stage is the CNN-based ResNet152 classifier model for benign or malignant skin lesions classification.

Intelligent ROI-based TL framework using CNN model with data augmentation is designed in (Ashraf et al., 2020). This reliable CAD system utilizes a k-means algorithm to identify accurate melanoma cancer from 
other types of skin cancer. Here, the images containing only the discriminative features of melanoma cells are used to train the model database of the system for high accuracy.

Deep Neural Networks (DNN) employing the Deep Learning (DL) algorithm promises medical image analysis (Namozov et al.,2018). Particularly, the classification of skin lesion images aided with adaptive activation functional linear units. This plays a significant role in skin image recognition for melanoma disease diagnosis. In this, the CNN network model with an adaptive method of functional activation outperforms the task of classifying the melanoma skin lesion.

The primary cause of skin cancer is deoxyribonucleic acid in skin cells that causes abnormalities and changes is explained in (Dildar et al., 2021). Various approaches for skin cancer detection are thoroughly examined in this study. The DNN model uses the DL method to extract malignant melanoma characteristics such as color, symmetry, form, and size from lesion parameters. The categorization of benign skin cancer and melanoma is then done using an effective SVM classifier.

Basal cell carcinoma is a skin cancer that is detected using image processing and Artificial Neural Network (ANN) method (Bayot et al., 2007). This study is from a small piece of the skin with the special characteristics of basal cells. Low power magnification shows the BCC detection, whereas high power magnification shows the dark cells.

A transformation method providing the wavelet approximation coefficients sequentially is the fastest diagnostic method of malignant melanoma and seborrheic keratosis lesion classification (Serte et al., 2019). This automated modeling of horizontal, approximate, and vertical wavelet coefficients is measured using the DWT employing DL techniques. TL-based ResNet-50 and ResNet-18network models using the skin images and their corresponding coefficients with more output probabilities are evaluated.

Malignant melanoma and seborrheic keratosis are accurately diagnosed using Gabor wavelet based DCNN (Serte et al., 2019). Feature extraction from seven-level decomposition of the input image into sub-band images is directly fed to eight parallel $\mathrm{CNN}$ classifier models to generate eight different probabilistic predictions. Finally, decomposed images are fused for image reconstruction using the sum rule and classify the skin lesion images.

The first stage is when CAD-based early diagnosis of malignant type skin cancer images are denoised using the median filter (Xu et al., 2020). The second stage is the CNN-based image segmentation utilizing Satin Bowerbird Optimization (SBO). Finally, the processed image is classified using the SVM classifierinto the cancerous and healthy skin images.

CNN's approach of visualization of NN node-link interactions forms the toy-like networks that form the static diagrams (Harley et al., 2015). This model overcomes the complex network issues by interacting using the drawing pad to visualize digit recognition. These activation patterns formed by the network respond to the realtime behavior of the user-given input.

A DL system equipped with a graphic processing unit for identifying melanoma lesion images is explained in (Nasr-Esfahani et al., 2016). The non-dermoscopic or clinical images that are obtained from the clinics are first denoised and enhanced using the filtering method of pre-processes. These enhanced images are stored in the pre-trained CNN-based DL system database. Finally, the CNN classifier model aids the classification of melanoma and normal images.

DCNN employing deep networks with appropriately 50 layers acquire more dominant discriminative features with more accuracy (Yu et al., 2016). First, the residual network with the DL technique degrades the overfitting problem as the network goes deeper. The technique ensures a high-performance network for feature extraction and a deep residual network classifier for efficient melanoma skin classification.

Among all computerized network, the CNN method is superior for skin lesion classification (Mahbod et al., 2019). This system employs optimized in-depth features from different abstraction levels of the hybrid model. AlexNet, VGG16, and ResNet-18 are the three pre-trained models utilizing the deep feature generators for feature extraction. Finally, the SVM classifier outputs are fused to obtain a deep hybrid method of classification.

Automatic skin lesion analysis is performed using DL techniques to classify skin dermoscopic images (Singh et al., 2018). Melanoma type skin cancer holds the most prevalent form of cancer features aids to diagnose using the DL-based DCNN classification system employing the random forest classifier model.The high prognosis rate is resulted from the early detection of skin cancer by identifying the abnormalities in the skin (Jacob et al., 2021). Since the shape and color of the abnormalities vary with sample images, the fractal model for skin cancer diagnosis is designed. The fractal features are obtained from the differential box counting method. 


\section{Proposed Methodologies}

In our study, the image acquisition with denoising and contrast enhancement is made using the median filter in the initial stage of pre-processing. With the help of wavelet transformation, using the multi-level representation of skin lesion image features is stored in the model database. Then the feature images obtained are fed to the simple convolutional layered VGG-16 architecture and employ the CNN classifier model to classify the system.

The architecture of the VGG16 network model utilizes the reduced convolution layer architecture using the TL approach with efficient classification. The flow of the proposed CNN-VGG-16 network model block diagram is shown in Fig.1, consisting of several steps, i.e., acquisition, increase of contrast, pre-processing, wavelet transformation, training/testing/validation of data set production, and the suggested methodology. Fig.1 shows the overview of proposed CNN-VGG-16 network model approach for wavelet transform.

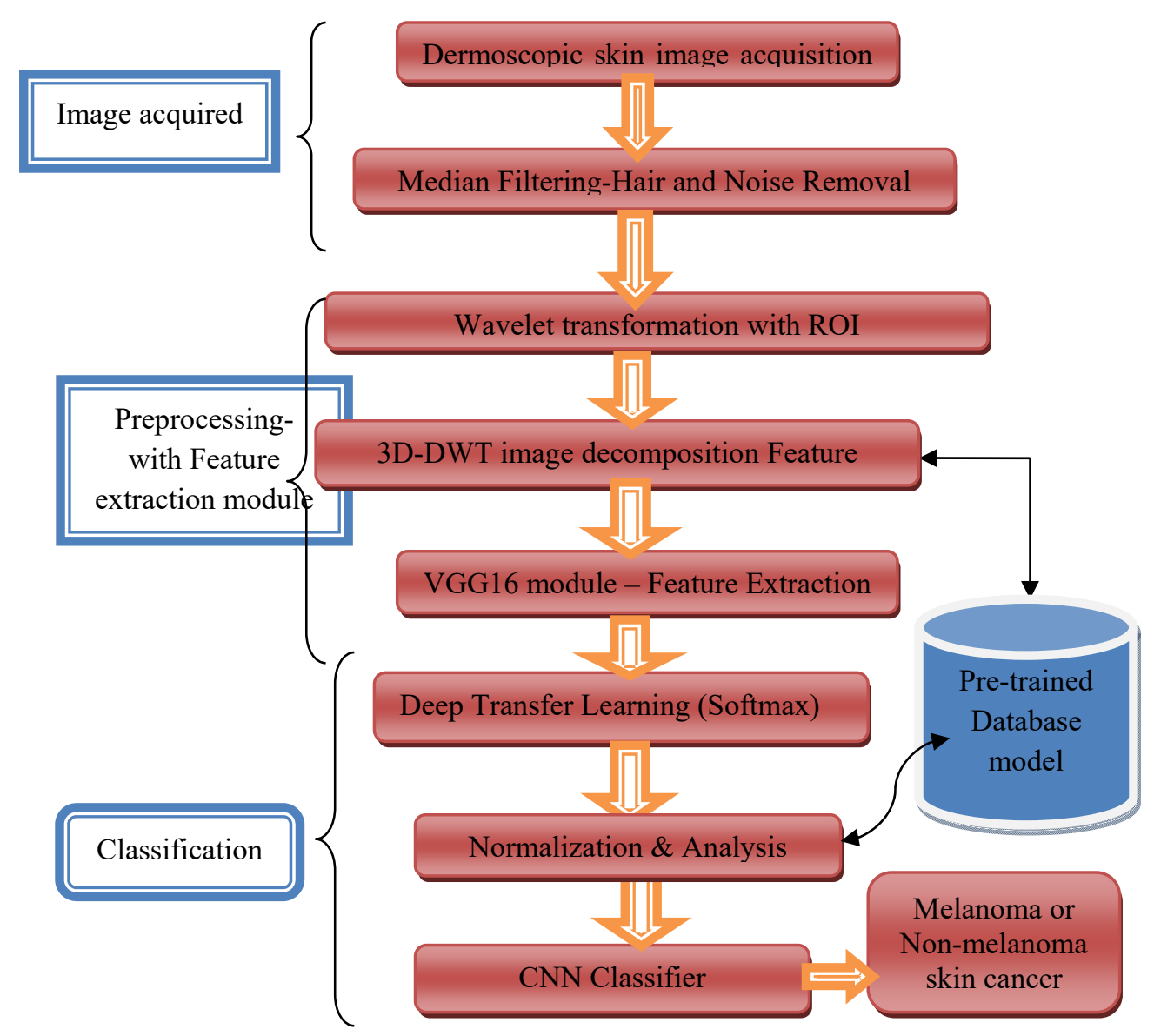

Fig.1. Flow of proposed Wavelet transformed CNN-VGG-16 network model

\subsection{Wavelet Feature Extraction}

The DWT decomposes a signal into multiple sets, each one is a time series of coefficients describing the signal's temporal variations in the corresponding frequency band. The 2D-DWT helps extract the twodimensional features, and the 3D-DWT aids in extracting the three-dimensionalwavelet features independently from skin images.

$$
\begin{aligned}
& C^{T_{\psi}(D, T)}=\int_{-\infty}^{\infty} x(t) \psi_{D, T}(t) d t \\
& \psi_{D, T}(t)=\frac{1}{\sqrt{D}} \psi\left(\frac{t-D}{T}\right)
\end{aligned}
$$


The Eq. (1) of $C^{T}$ is used to discrete DWT transformation using dilation and time.Eq (3) is the approximate components and Eq. (4), represents the detailed components.

$$
\begin{aligned}
& \operatorname{Approx}_{s, k}(n)=\downarrow\left[\sum_{n} x(n) L_{s}^{*}\left(n-2^{s} k\right)\right] \\
& \operatorname{Detail}_{s, k}(n)=\downarrow\left[\sum_{n} x(n) a H_{s}^{*}\left(n-2^{s} k\right)\right]
\end{aligned}
$$

The CWT provides time-frequency resolution, and this multiresolution may be achieved using filter banks to generate the DWT. The filter bank's low-pass $(L p(n))$ and high-pass $(H p(n))$ filtering branches retrieve approximation and details of the skin images, respectively. Fig. 2 represents the 1D and 2D DWT-level filter bank. Depending on the desired resolution, the filter bank can be extended to any length. The down sampling doubles the frequency resolution, and the coefficient represents the lowest half of the frequencies.

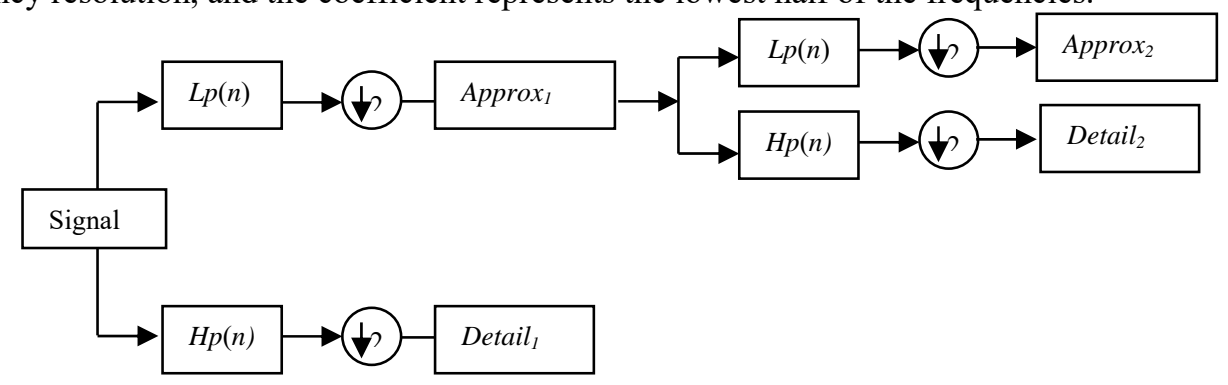

Fig.2 (a). 1D-DWT (signal)

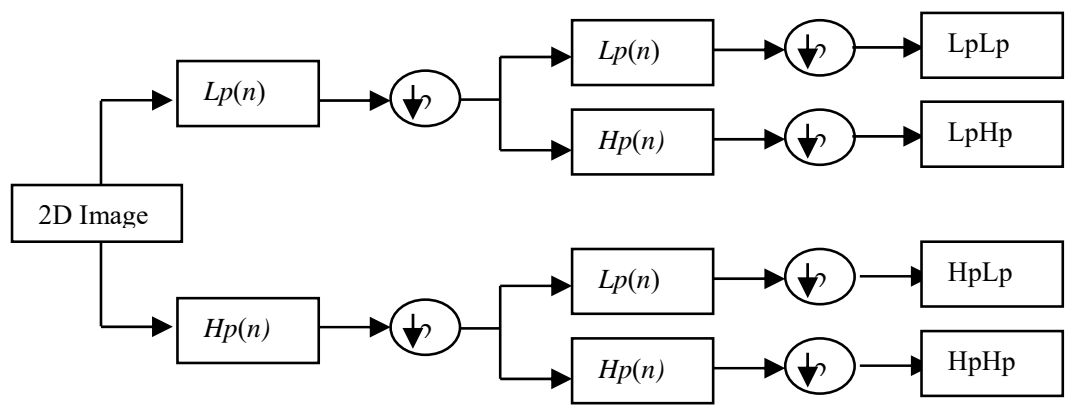

Fig. 2(b). 2D-DWT (image)

Because dermoscopic images are color images saved in RGB format, the input color images must first be transformed to grayscale before features can be retrieved. The 2D-DWT decomposed and retrieves sub-images are shown in Fig. 3.

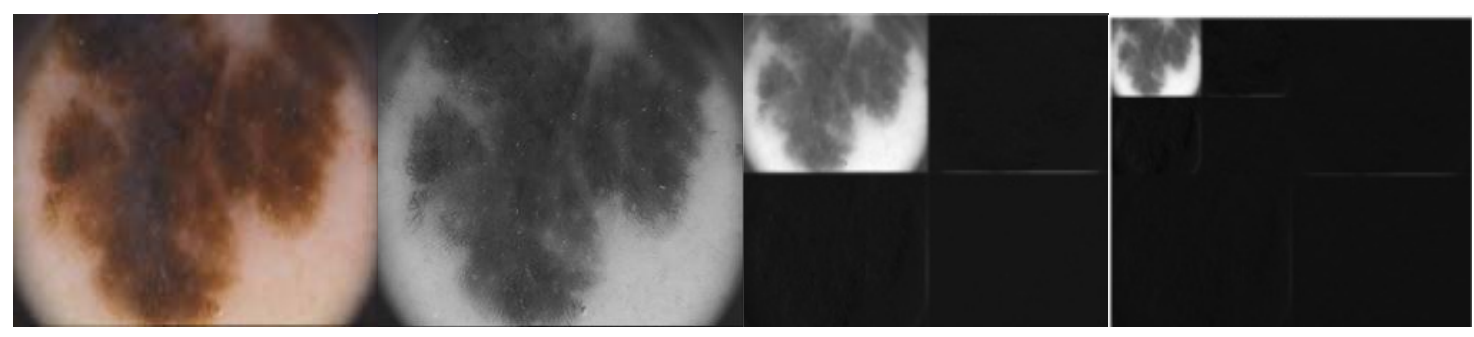

Fig. 3. Sample skin image: grayscale image, $1^{\text {st } l e v e l ~ D W T, ~} 2^{\text {nd }}$ level decomposition

The images are the RGB color images features; that are considered as input for three-dimensional or 3level wavelet transformation of the skin image. Hence, to extract the color images directly from texture information, 3D-DWT is used. Fig. 4 shows the 3D-DWT feature extraction of the RGB skin image. 


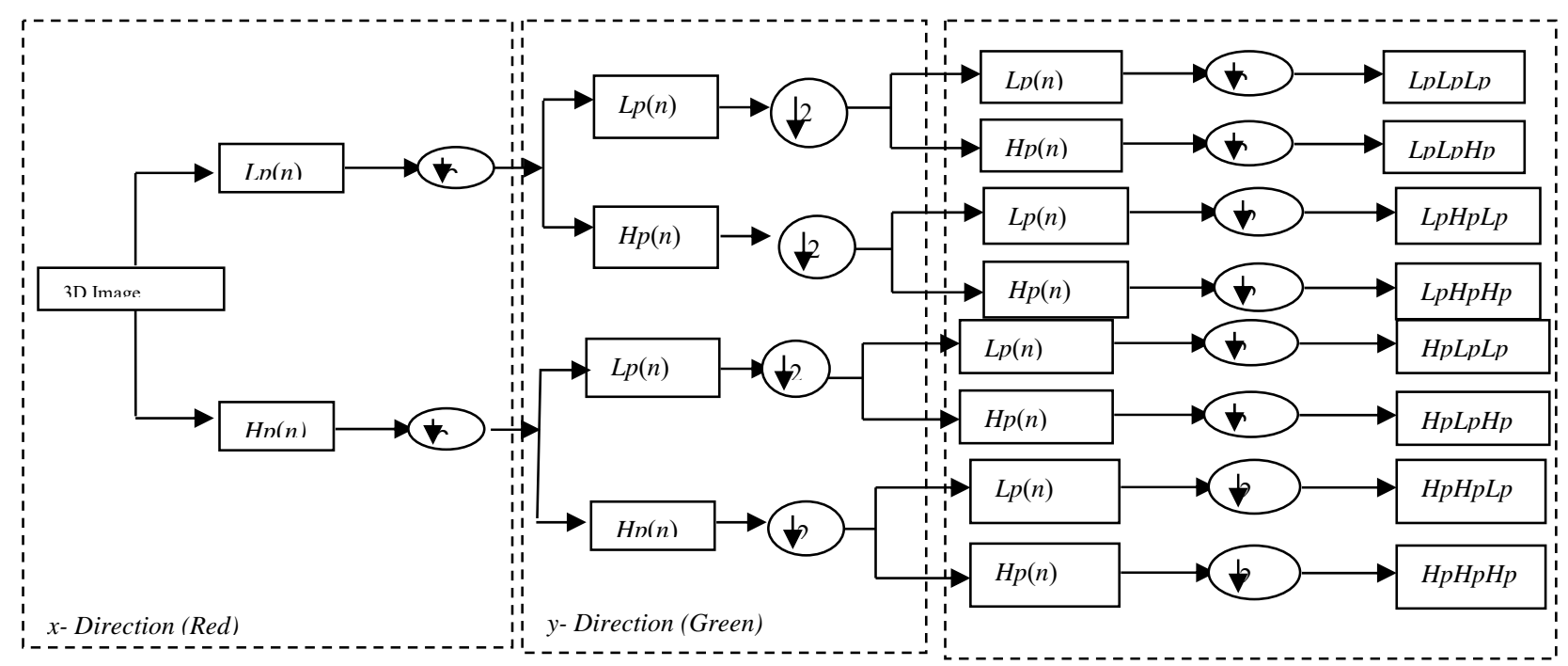

Fig. 4. 3D-DWTmulti-level feature extraction from RGB

Table 1 shows the DWT decomposition levels for two dimensional and three dimensional and its corresponding sub-band levels.

\begin{tabular}{|c|c|c|}
\hline $\begin{array}{c}\text { Decomposition } \\
\text { Levels }\end{array}$ & $\begin{array}{c}\text { 2D-DWT sub } \\
\text { band }\end{array}$ & $\begin{array}{c}\text { 3D-DWT sub } \\
\text { band }\end{array}$ \\
\hline 1 & 4 & 16 \\
\hline 2 & 7 & 22 \\
\hline 3 & 10 & 29 \\
\hline 4 & 13 & 36 \\
\hline 5 & 16 & 43 \\
\hline
\end{tabular}

Table 1. Number of sub-bands by 2D-DWT and 3D-DWT

Following decomposition, the features are retrieved using the normalization method the subbands ranging from [ 0255$]$ and the co-ocuurence matrix for the normalised subbands are computed. They are represented by the letter $\mathrm{n}$ and the features extracted as follows:

1. the contrast features aids in computing the local variance using the Eq. (1).

$$
\sum_{i, j}|i-j|^{2} n(i, j)^{2}
$$

2. Correlation features compute the texture's relations by Eq.(2).

$$
\sum_{i, j} n_{i j} \frac{(i-\mu)(j-\mu)}{\sigma^{2}}
$$

3. The energy features compute the texture's energy by Eq. (3).

$$
\sum_{i, j} n(i, j)^{2}
$$

4. The homogeneity features compute the texture's uniformity by Eq. (4).

$$
\sum_{i, j} \frac{n(i, j)}{1+|i-j|}
$$

For each sub-band, these extracted features are stored for further analysis. Table 2 shows the number of features extracted. In the testing phase, the dermoscopic image feature extraction is performed, and then the 3DDWT is predicted efficient from the results obtained in Table 2. 


\begin{tabular}{|c|c|c|}
\hline & \multicolumn{2}{|c|}{ Features Extracted } \\
\hline $\begin{array}{c}\text { Sub band } \\
\text { levels }\end{array}$ & $\begin{array}{c}\text { 2D-DWT } \\
\text { approach }\end{array}$ & $\begin{array}{c}\text { 3D-DWT } \\
\text { approach }\end{array}$ \\
\hline 1 & 16 & 64 \\
\hline 2 & 28 & 88 \\
\hline 3 & 40 & 116 \\
\hline 4 & 52 & 144 \\
\hline 5 & 64 & 174 \\
\hline
\end{tabular}

Table 2. Number of Features extracted

Hence, in this study, 3D-DWT is adopted and that depends on the degree of expression. This affects the performance and must be modified to provide the optimal performance measurements. To achieve this, a CNNbased deep TL method extracting features from altered skin images is used.

\subsection{Convolutional Neural Network (CNN) model}

An NN is a nonlinear and statistical prediction approach. The artificial NN is modeled after the human neural network. Convolutional Neural Networks are the linkages (CNN). The input layer sends data to the first of three layers of neurons. The hidden layers are the mid-layers, where the medium neurons send information to the third neuron layer. The number of layers hidden in the CNN varies according to input images, as the dataset interconnects the first layer to the hidden layer. The data collection can be labeled and processed using a supervised or unsupervised learning method suitablyuses the TL method to learn hidden characteristics, as shown in Fig. 5.

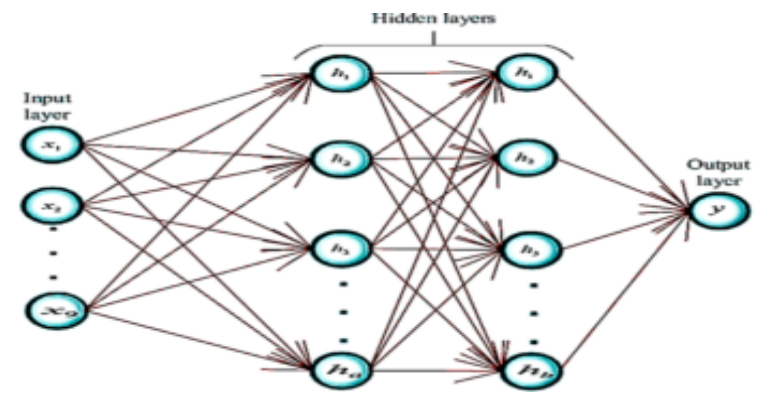

Fig. 5. CNN architecture with hidden layers

\subsection{Transfer Learning}

Our study may employ a TL method, an efficient method providing the high-resolution skin image output of the 3D-DWT technique. The VGG-16 network model utilizes the CNN-based TL approach.

\subsubsection{VGG-16 Architecture with reduced complexity employing CNN-based classification Techniques}

The network model uses VGG-16.CNN is a basic form of deep neural network that is widely used. It's used to classify images, put together a group of image pixels, and identify images. In this study, we suggested a basic model of RGB Color channels with a batch size of 32 using our CNN model, which extracts the suspicious region as $224 \times 224$ input sizes. $\mathrm{CNN}$ is an excellent technique for collecting and learning global and local data by combining simple features like curves and edges to generate more complex features like shapes and corners. Convolution layers, with nonlinear max-pooling layers and fully connected (FC) layers, are some of CNN's hidden layers are shown in Fig. 6.
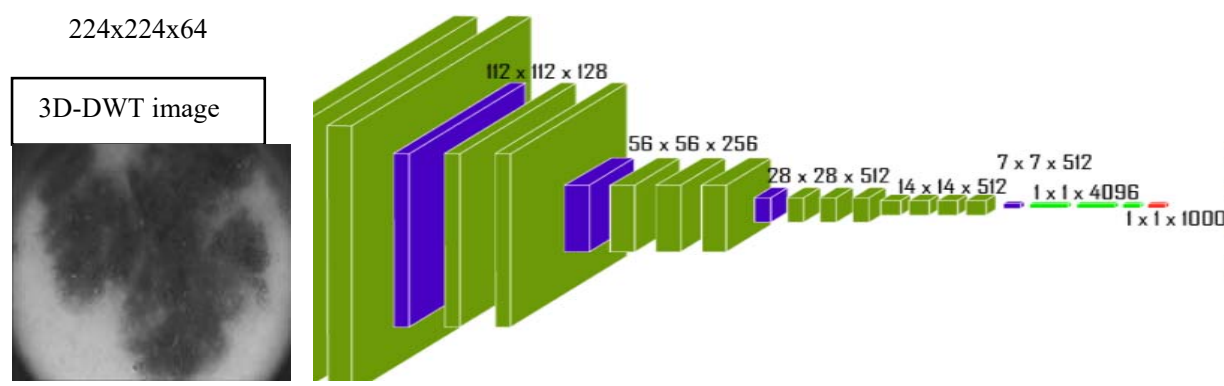

convalution + ReLL

max pooling

fully Connected + ReLU

softmax

Fig.6. VGG-16 network with CNN-based model

In Fig. 6, the proposed VGG-16 network architecture with FC and SoftMax is depicted with grey skin images. The architecture has 64 filters and doubles 128, 256, and 512 filters in the final layers. The architecture 
comprises 16 convolution layers with a fixed filter size $3 \times 3$ and five max-pooling layers of $2 \times 2$-size across the network. And the top two layers with the output layer is the SoftMax.

\section{Results and Discussion}

The proposed system model is examined, and the performance is measured using 200 dermoscopic skin images obtained from the $\mathrm{PH}^{2}$ database [15]. Using a CNN-based classifier system, the recovered VGG-16 network features are classified as normal and abnormal skin image classes.The images in the database are categorized into two classes. The normal images are classified in the first class, as illustrated in Fig. 7(a).

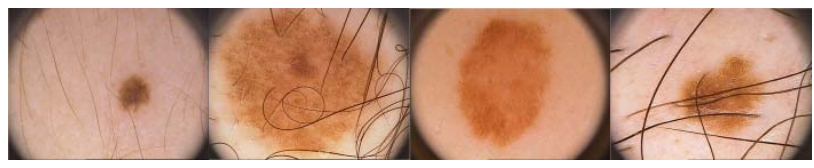

Fig 7(a). Normal skin images

The second category is the abnormal images that are benign (non-melanoma cancer), and melanomas (malignant cancer) samples from the database are shown in Fig. 7(b).

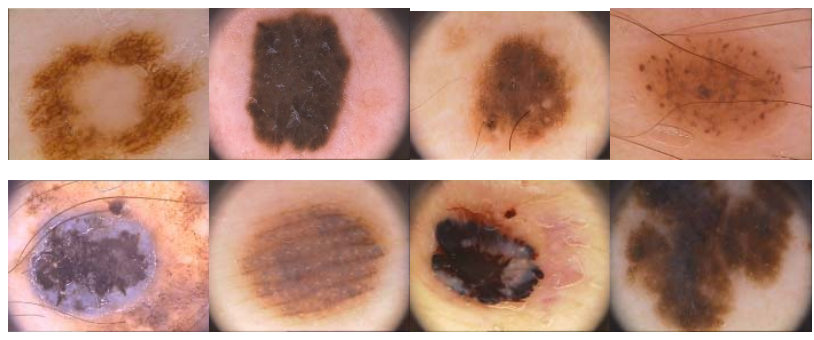

Fig. 7(b). Abnormal skin images- Benign cancer (top row) and Malignant cancer (bottom row)

For evaluating, the confusion matrix using $\mathrm{k}$-fold cross-validation, with $\mathrm{k}=10$, is used. The performance metrics accuracy, sensitivity, and specificity are determined using Eq. (9) to (11) based on the results of the cross-fold validation.

$$
\begin{gathered}
\text { Accuracy }=\frac{T P+T N}{T P+F N+T N+F P} \\
\text { Sensitivity }=\frac{T P}{T P+F N} \\
\text { Specificity }=\frac{T N}{T N+F P}
\end{gathered}
$$

The DWT levels of feature extraction and its performance is measured. To explore the relevance of the proposed system's pre-processing and feature selection components, the entire system run similarly utilizing 5 levels 3DWT. The results of their categorization are then computed and presented in Table 3.

\begin{tabular}{|c|c|c|c|c|c|c|}
\hline \multirow{2}{*}{$\begin{array}{c}\text { DWT sub } \\
\text { band } \\
\text { Levels }\end{array}$} & \multicolumn{3}{|c|}{ 2D-DWT } & \multicolumn{3}{c|}{ 3D-DWT } \\
\cline { 2 - 6 } & Accuracy & Sensitivity & Specificity & Accuracy & Sensitivity & Specificity \\
\hline $\mathbf{1}$ & 85.48 & 76.77 & 85.48 & 86.25 & 91.33 & 91.33 \\
\hline $\mathbf{2}$ & 89.33 & 82.92 & 89.33 & 90.00 & 93.67 & 93.67 \\
\hline $\mathbf{3}$ & $\mathbf{9 3 . 0 0}$ & $\mathbf{8 9 . 1 7}$ & $\mathbf{9 3 . 0 0}$ & $\mathbf{9 5 . 4 2}$ & $\mathbf{9 7 . 0 0}$ & $\mathbf{9 7 . 0 0}$ \\
\hline $\mathbf{4}$ & 91.00 & 85.83 & 91.00 & 92.08 & 95.00 & 95.00 \\
\hline $\mathbf{5}$ & 90.00 & 84.17 & 90.00 & 88.75 & 92.67 & 92.67 \\
\hline
\end{tabular}

Table 3. Performance measures of DWT levels

The confusion matrices show the various predictions based on the probability of outcome and are shown in Fig. 8. The outcomes are predicted as TP, TN, FP, and FN. 

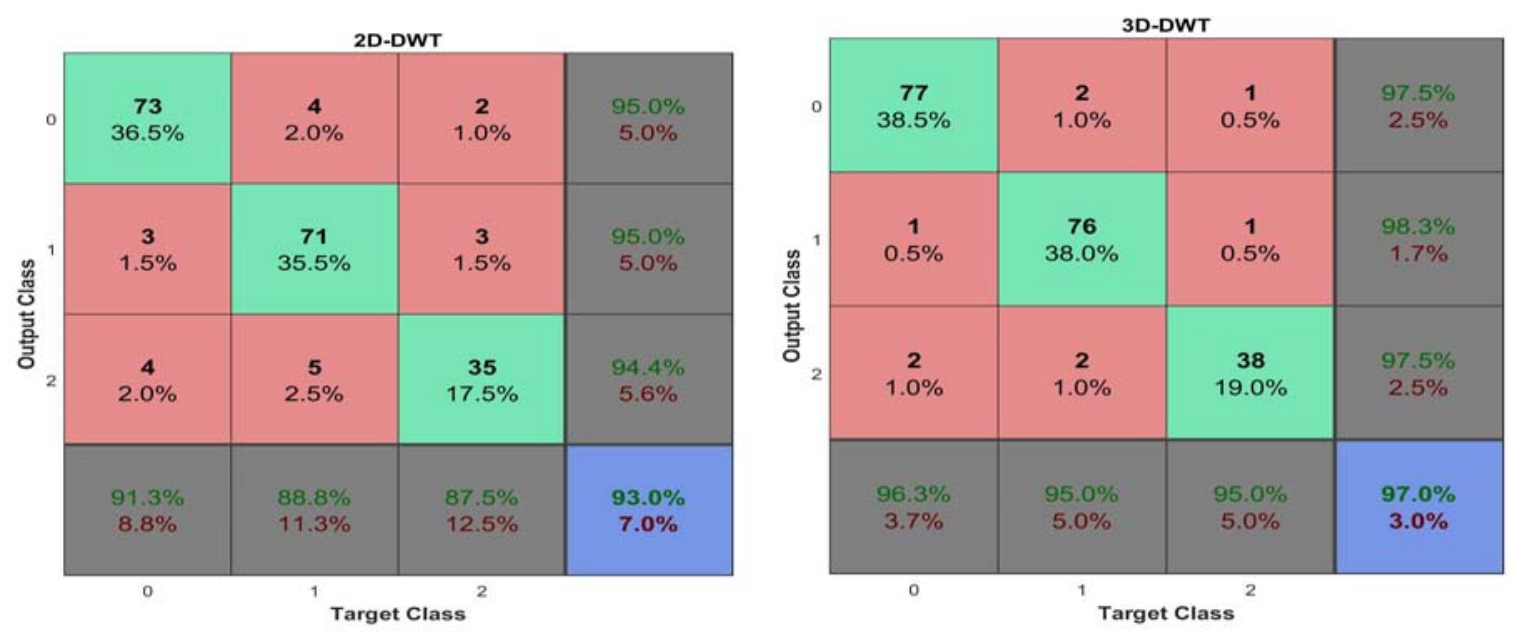

Fig. 8. Performance measure of 2D-DWT and 3D-DWT using a confusion matrix.

From Table 3, it is observed that the feature extracted from the $3^{\text {rd }}$ level provides COFs at higher levels with more promising effects. With hybrid features, the SCDS achieves a maximum accuracy of $93 \%$, compared to $78 \%$ and $84 \%$ for their separate counterparts. SCDS has a sensitivity of $91.67 \%$ and a specificity of $95 \%$ for the same feature sets. Furthermore, regardless levels indicates thatskin lesions are properly identified more often than abnormal images.

\section{Conclusion}

Although the VGG16 convolutional net consists of outstanding architecture, we still need to look for over compatibility where the smaller images might have produced a sample overlaying in a feature space. The testing image ROI features extracted from the VGG-16 Net are classified using the CNN classifier. The extracted features are used VGG16 convolution network tuning is very efficient, and the CNN classifier employed achieves more accuracy. The textural feature evaluation is done. The usage of wavelet coefficient is frequently regarded as an essential technique. The value provides a novel skin cancer detection. This system assesses using the impact of textural characteristics. Deep learning methods withpre-processing step removes hairs and noises from dermoscopic images that increase the SCDS system's performance in the future. The $\mathrm{PH}^{2}$ database dermoscopic images are used to test the co-occurrence feature-based derived from two-dimensional and threedimensional wavelets. The results demonstrate that these features work well in a hybrid method providing $93 \%$, $91.67 \%$ and $95 \%$ for accuracy, sensitivity, and specificity respectively.

\section{References}

[1]. Acosta, M. F. J.; Tovar, L. Y. C.; Garcia-Zapirain, M. B.; Percybrooks, W. S. (2021): Melanoma diagnosis using deep learning techniques on dermatoscopic images. BMC Medical Imaging, 21(1), pp. 1-11.

[2]. Ashraf, R.; Afzal, S.; Rehman, A. U.; Gul, S.; Baber, J.; Bakhtyar, M.; Maqsood, M. (2020): Region-of-Interest based transfer learning assisted framework for skin cancer detection. IEEE Access, 8, pp. 147858-147871.

[3]. Bayot, A. R.; Samoy, L. A. M.; Santiago, N. A. D.; Tomelden, M. R. Sybingco, E. (2007): Malignancy detection of candidate for basal cell carcinoma using image processing and artificial neural network, Dlsu Eng. E J., 1, pp. 70-79.

[4]. Dildar, M.; Akram, S.; Irfan, M.; Khan, H. U.; Ramzan, M.; Mahmood, A. R. Mahnashi, M. H. (2021): Skin Cancer Detection: A Review Using Deep Learning Techniques. International journal of environmental research and public health, 18(10), pp. 5479.

[5]. Harley, A. W. (2015). An interactive node-link visualization of convolutional neural networks. In International Symposium on Visual Computing, pp. 867-877

[6]. Jacob, S.; Rosita, J. D. (2021): Fractal model for skin cancer diagnosis using probabilistic classifiers. International journal of advances in signal and image sciences, 7(1), pp. 21-29.

[7]. Mahbod, A.; Schaefer, G.; Wang, C.; Ecker, R.; Ellinge, I. (2019): Skin lesion classification using hybrid deep neural networks. IEEE International Conference on Acoustics, Speech and Signal Processing, pp. 1229-1233.

[8]. Namozov, A.; Ergashev, D.; Im Cho, Y. (2018): Adaptive activation functions for skin lesion classification using deep neural networks. International Conference on Soft Computing and Intelligent Systems, pp. 232-235.

[9]. Nasr-Esfahani, E.; Samadi, S.; Karimi, N.; Soroushmehr, S. M. R.; Jafari, M. H.; Ward, K.; Najarian, K. (2016): Melanoma detection by analysis of clinical images using convolutional neural network. IEEE International Conference on Engineering in Medicine and Biology Society, pp. 1373-1376.

[10]. Serte, S.; Demirel, H. (2019): Wavelet-based deep learning for skin lesion classification. IET Image Processing, 14(4), pp. 720-726.

[11]. Serte, S.; Demirel, H. (2019): Gabor wavelet-based deep learning for skin lesion classification. Computers in biology and medicine, 113, pp. 103423.

[12]. Singh, V.; Nwogu, I. (2018): Analyzing skin lesions in dermoscopy images using convolutional neural networks. IEEE International Conference on Systems, Man, and Cybernetics, pp. 4035-4040.

[13]. Xu, Z.; Sheykhahmad, F. R.; Ghadimi, N.; Razmjooy, N. (2020): Computer-aided diagnosis of skin cancer based on soft computing techniques. Open Medicine, 15(1), pp. 860-871.

[14]. Yu, L.; Chen, H.; Dou, Q.; Qin, J.; Heng, P. A. (2016): Automated melanoma recognition in dermoscopy images via very deep residual networks. IEEE transactions on medical imaging, 36(4), pp. 994-1004.

[15]. $\mathrm{PH}^{2}$ Database Link: https://www.fc.up.pt/addi/ph2\%20database.html 


\section{Authors Profile}

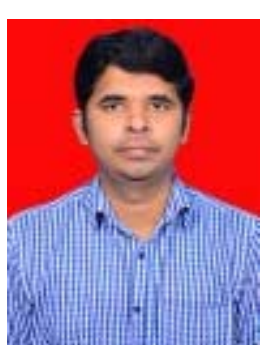

Mr. S. P Maniraj is presently working as Assistant Professor in Computer Science and Engineering at SRM Institute of Science and Technology. He received Master of Technology in Embedded System Technologies during 2008-10 from Anna University Tirunelveli and also received Bachelor of Engineering in Computer Science and Engineering during 2004-2008 from Anand Institute of Higher Technology. Presently he is pursuing Ph.D. in the field of Image Processing from Sathyabama Institute of Science and Technology, Chennai, India.

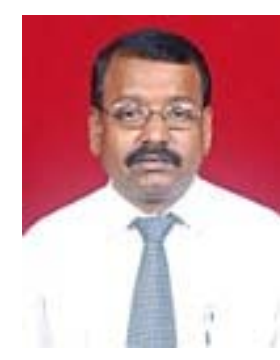

Dr. P. Sardar Maran is presently working as Associate Professor in the Department of Computer Science \& Engineering, Sathyabama Institute of Science and Technology ,Chennai., India. He received Ph.D from Sathyabama Institute of Science and Technology in 2015. He has more than 30 publications in reputed International Journals and has participated and presented papers in several National and International conferences. His research interest includes Artificial Intelligence, Machine Learning, Deep Learning, Image Processing and Computer vision. 